\title{
'Second look' operation for ovarian dysgerminoma
}

\author{
Harold Ellis \\ D.M., M.Ch., F.R.C.S.
}

\author{
T. A. M. StOKeR \\ M.D., F.R.C.S.
}

Surgical Unit, Westminster Hospital, London, S.W.1

\section{Introduction}

Ovarian dysgerminomas are uncommon tumours and only five cases have been treated over the past 20 years at Westminster Hospital. It is estimated that they represent approximately $1 \%$ of all ovarian cancers (Mueller, Topkins \& Lapp, 1950). Since their original description by Mayer in 1931 they have teen recognized as tumours of considerable malignant potential with capabilities of local invasion and extensive distant metastases both via the lymphatics and the blood stream. Macroscopically the tumour is solid, homogeneous, rubbery and white, although the cut surface may be yellowish due to lipoid degeneration. The tumour may reach considerable size and is frequently bilateral. Microscopically dysgerminoma closely resembles a seminoma with large polyhedral cells arranged in cords in a fibrous stroma of variable density and with a variable degree of lymphocytic infiltration. Although the tumour closely resembles seminoma of the testis (in the past it has been called seminoma ovarii) it has no endocrine effects although there is an associated incidence of pseudo-hermaphroditism. The majority of cases occur in adolescence and young adults, although patients as young as 2 years and as old as 76 years have been recorded. It appears that the prognosis is worse in those under 15 years of age (Pedowitz, Felmus \& Grazzel, 1955).

Published reports show that there is considerable variation in the malignancy of dysgerminomas, varying from a perfectly benign course to considerable agressiveness, but overall their prognosis is better than that of seminoma of the testicle in spite of the virtually identical histological appearance. Mueller et al. (1950) estimated a 5-years survival rate of $27 \%$ with three-quarters of those dying from the disease doing so within the first year after diagnosis. However, Thoeney et al. (1961) in a study of twenty-seven cases reported a 70\% 5-year survival.

We here report a child who has undergone a successful 'second look' block dissection of invaded abdominal lymph nodes 2 years after initial removal of the primary tumour.

\section{Case report}

The child was first seen at the age of 8 years with a month's history of central abdominal pain. Examination revealed a nodular mass arising from the pelvis and extending into the left iliac fossa. X-rays of chest and abdomen, full blood count and ESR were normal. A barium enema showed the sigmoid colon to be displaced upwards and to the right. Laparotomy was carried out on 4 January 1966 (H.E.). A hard nodular tumour of the left ovary was exposed which measured $11 \mathrm{~cm}$ in diameter (Fig. 1). It was adherent to the posterior surface of the uterus and to both broad ligaments and there was loculated mucus material attached to the ovary. The right ovary was clinically normal and there was no evidence of spread of the disease to the lymph nodes or to the liver. A frozen section was reported as being consistent with dysgerminoma and in consequence of this a total hysterectomy with bilateral salpingo-oophorectomy was performed. Recovery was uneventful. Histological examination of the specimen confirmed the diagnosis of dysgerminoma invading the broad ligament but the uterus and right ovary were clear of growth.

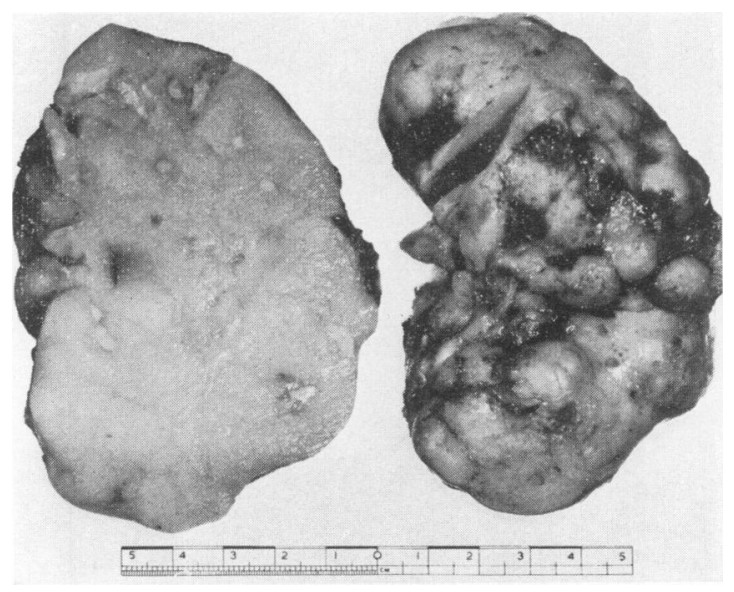

Fig. 1. Left ovarian tumour. 
Postoperatively the child was treated on the $6 \mathrm{mV}$ linear accelerator to anterior and posterior pelvic fields measuring $9 \times 13 \mathrm{~cm}$ to a total tumour dose of $3300 \mathrm{r}$.

The patient remained well on close clinical supervision and serial chest $\mathrm{X}$-rays were also completely normal. However, a routine X-ray of the abdomen in January 1968 showed calcification in a rounded mass which was considered to represent a metastasis in a para-aortic lymphatic gland. Because of this, a lymphangiogram was carried out (Fig. 2) which showed good filling of the vessels and the inguinal iliac and para-aortic nodes on both sides. No abnormality was seen with the exception of the rounded calcified lymph node to the left of the vertebral column.

Because of the presumed diagnosis of secondary deposits in the para-aortic nodes, further laparotomy was decided upon. Clinical examination was negative and, once again, chest X-ray, full blood count and liver function tests were also with normal limits.

A 'second look' laparotomy was carried out on 16 February 1968 (H.E.). A left paramedian incision was made and both the liver and pelvis found to be clear of disease. There was a mass of involved lymph nodes on the left posterior abdominal wall

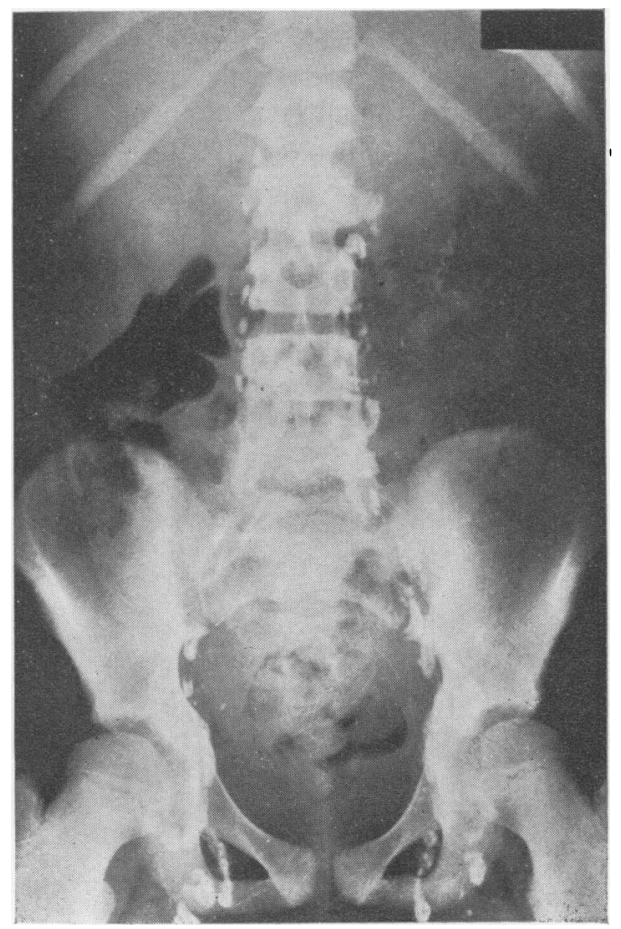

Fig. 2. Lymphangiogram.

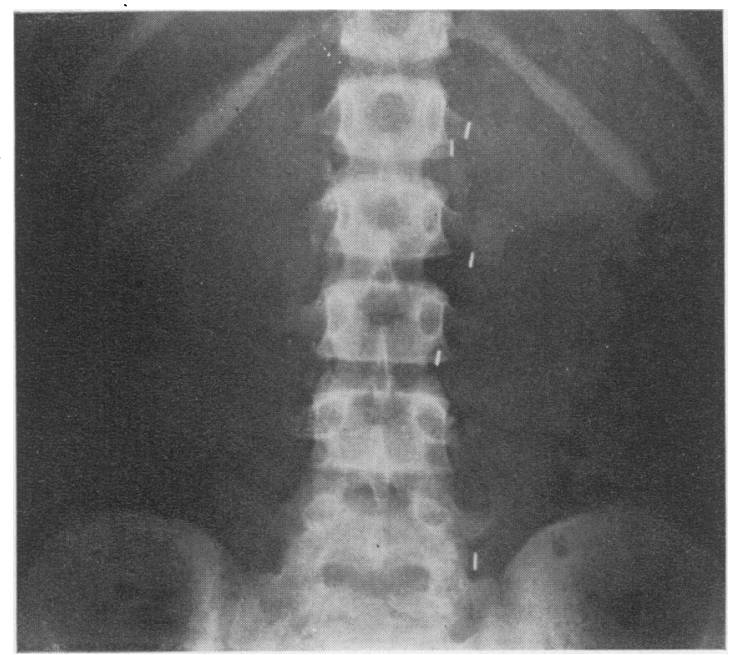

FIG. 3. X-ray of abdomen showing limits of excision of retroperitoneal tumour.

extending from the brim of the pelvis to the level of the splenic vein. The descending colon was mobilized to the left, the left ureter identified and freed and an en bloc removal of the left retroperitoneal lymph nodes performed. The limits of excision weres marked with Cushing clips (Fig. 3).

Postoperative recovery was uneventful.

Histological examination showed that the lowermost node revealed evidence of lymphangiography but not of metastatic disease. The rest of the excised specimen showed metastatic dysgerminoma and one node demonstrated complete necrosis of tumour tissue with calcification.

Postoperatively, the left para-aortic region was treated on the $6 \mathrm{mV}$ linear accelerator to a total dose of $3500 \mathrm{r}$.

The patient has remained well and free from evidence of recurrence both clinically and on X-rays of the chest and abdomen. She is now 12 years of age and is receiving ethinyloestradiol $0.1 \mathrm{mg}$ b.d.

\section{Discussion}

Primary treatment of dysgerminoma is normally by a combination of surgery and radiotherapy although it appears that some examples may exhibit hormone-dependence since Russo \& Kelso (1949) reported an example in which tumour regression recurred following male hormone therapy. It is usually agreed that the primary tumour is best treated by total hysterectomy and bilateral salpingooophorectomy followed by postoperative radiotherapy. Procedures designed to preserve child bearing capacity usually fail (Pedowitz et al., 1955). Dysgerminomas are well recognized as being highly radiosensitive and even examples with apparently 
very poor prognosis may respond well to this treatment. Thus, Gruss (1932) described a patient alive and well 9 years after irradiation of extensive metastases and Dunster reported two cases with lymph node metastases who survived more than 5 years after local excision and radiotherapy (Dunster \& Bennet, 1953).

Spread of the tumour beyond the ovarian capsule, the presence of ascites or lymph node metastases are generally recognized as poor prognostic features. In our own patient, infiltration of adjacent pelvic structures was already present at the time of initial treatment which was by conventional radical surgery followed by radiotherapy. When there was obvious evidence radiologically of extensive metastases in the left para-aortic nodes it was considered, in consultation with our radiotherapist, Dr Kenneth Newton, that attempted removal of this extensive tumour mass surgically, rather than relying on radiotherapy appeared advisable. The patient underwent what Gilbertson \& Wangensteen (1962) have described as a symptomatic 'second look operation' when it had become apparent that metastases were present. Should her tumour-free state continue, she may also exemplify the fact that delayed regional lymphadenectomy, analagous to that advocated by Crile (1966) may frequently be effective in ablation of the tumour.

For many years now, Wangensteen \& Sosin (1968) has advocated a 'second look' policy for malignant tumours of the colon, stomach, ovary and retroperitoneum. In this programme, apparently curative resections in which lymph nodes are found to be histologically involved are followed 6 months later by further exploration. If recurrent malignant disease is found this is excised and re-exploration performed in a further 6 months. The programme is continued until either there is no further evidence of recurrent disease or until unresectable recurrences are detected. With this policy he has shown that some $10 \%$ of patients with residual tumour can be converted to a tumour-negative state by repeated surgery. It is easy to take issue with a policy of second look operations in patients who are asymp- tomatic and clinically disease-free. Objections include the tremendous extra burden on already strained hospital services and the necessity of detailed explanation of the situation to the patient in order to get him to consent to further laparotomy. There seems little doubt, however, that in selected cases clinical or radiological evidence of recurrent disease may be followed by prolonged remissions and this has been our policy for some years (Cronin \& Ellis, 1958). The present case is advanced as an example of this policy.

\section{Acknowledgments}

We would like to thank Professor Alan Morgan for the histology report and the Department of Medical Illustrations, Westminster Hospital, for the photographs.

\section{References}

CrILE, G. (1966) Metastases from involved lymph nodes after removal of various primary tumours. Annals of Surgery, 163, 267.

Cronin, K. \& Ellis, H. (1958) The problem of suspected enuresis in cancer of the alimentary tract. British Medical Journal, 2, 1319.

Dunster, M. \& BENNET, D. (1953) The prognosis of dysgerminoma of the ovary. Journal of Obstetrics and Gynecology of the British Empire, 60, 402.

Gruss, J. (1932) Seminom (solides) grosszelliges Keimepithelblastom (des ovarium). Zentralblatt für Gynäkologie, 56, 2397.

Gilbertson, V.A. \& Wangensteen, O.H. (1962) A summary of 13 years experience with the second look programme. Surgery, Gynecology and Obstetrics, 114, 438.

Mueller, C.W., Topkins, P. \& LaPP, W.A. (1950) Dysgerminoma of the ovary: an analysis of 427 cases. American Journal of Obstetrics and Gynecology, 60, 153.

Pedowitz, P., Felmus, L.B. \& Grazzel, D.M. (1955) Dysgerminoma of the ovary; prognosis and treatment. American Journal of Obstetrics and Gynecology, 70, 1284.

Russo, P.E. \& Kelso, J.W. (1949) Dysgerminoma-a case with pulmonary metastases; result of treatment with irradiation and male sex hormone. Radiology, 52, 367.

Thoeney, R.H., Docherty, M.B., Hunt, A.B. \& Childs, D.S. (1961) A study of ovarian dysgerminoma with emphasis on the role of radiation therapy. Surgery, Gynecology and Obstetrics, 113, 692.

WANGENSTEEN, O.H. \& Sosin, H. (1968) How can the outlook in alimentary cancer be improved? American Journal of Surgery, 115, 7. 